\section{Vitreoretinal surgery for shotgun eye injuries: outcomes and complications}

Z Khoueir ${ }^{1}$, G Cherfan $^{1}$ and A Assi ${ }^{1,2}$

\begin{abstract}
Purpose To analyse the postoperative anatomic and functional outcomes in addition to complications after vitreoretinal surgery for patients with shotgun eye injuries related to hunting accidents.

Materials Retrospective review of the clinical records of all cases of shotgun eye injuries presented between January 2000 and January 2011 and with a minimum follow-up of 1 year. Collection of demographics, type of injury, choice of management, complications and final surgical success with final visual acuity is reported.

Results Twenty eyes of 19 patients (all male) with a mean age of 36.1 years (range 16-60 years) were included in the study. Mean postoperative follow-up was 47.5 months (range 15-118 months). Best corrected visual acuity (BCVA) at presentation ranged from perception of light to $20 / 200$. Ten eyes had a penetrating injury and 10 others had a perforating injury. All the eyes underwent an initial vitrectomy and the intraocular pellet was removed in all the 10 penetrating injuries. Concurrent cataract surgery was performed in 12 cases, internal tamponade was used in $\mathbf{1 5}$ cases and a supplemental encircling scleral buckle was

Introduction

Ocular trauma is still one of the major causes of preventable blindness and visual impairment occurring most commonly in children and young adults. ${ }^{1-5}$ According to the United States Eye Injury Registry Database BB and pellet guns account for nearly $6 \%$ of all ocular injuries and they still remain the most frequent gun injuries in the emergency setting. ${ }^{6,7}$ Although airsoft and BB gun-related injuries have been extensively investigated and reported, the literature is relatively sparse regarding pellet gun injuries to the eye and orbit. ${ }^{1,2,8-10}$ Earlier reports have insisted on the severity of this type of trauma and the necessity to reduce their incidence by publicising their risk and encouraging prophylactic measures such as eye protection. ${ }^{11-15}$ Experimental and clinical studies demonstrated that perforating injuries with posterior segment complications have a poor prognosis especially when treated by conventional techniques. ${ }^{16-18}$ The purpose of this study is to analyse the postoperative anatomic and functional outcomes in addition to complications after vitreoretinal (VR) surgery for patients with pellet gun eye injuries related to hunting accidents.
\end{abstract} inserted in 12 cases. One additional vitreoretinal surgery was required in seven cases $(35 \%)$ and two additional surgeries required in two other cases $(\mathbf{1 0} \%)$. At last follow-up BCVA ranged from NPL to 20/20 and was $20 / 100$ or better in 10 eyes $(50 \%)$. All patients had a flat retina except for two cases $(\mathbf{1 0} \%)$ that developed severe proliferative vitreoretinopathy.

Conclusion These results suggest that vitreoretinal surgery can offer good visual rehabilitation in patients with shotgun eye injuries.

Eye (2015) 29, 881-887; doi:10.1038/eye.2015.46; published online 1 May 2015

\section{Methods}

The medical records of all 19 consecutive patients presenting with pellet gun injuries to the posterior segment of the eye, and operated on between January 2000 and January 2011 and with a minimum follow-up of 1 year were reviewed retrospectively. All surgeries were done at the Beirut Eye Specialist Centre by two surgeons (AA and GC). Collection of demographics, type of injury, choice of management, complications, requirement for further surgery and final anatomical and functional outcomes are reported.
${ }^{1}$ Beirut Eye Specialist Hospital, Beirut, Lebanon

${ }^{2}$ Beirut Eye Clinic, Beirut, Lebanon

Correspondence:

Z Khoueir, Beirut Eye Specialist Hospital,

Al-Mathaf Square, PO Box 116-5311, Beirut, Lebanon Tel: +961 1 423111; +961 171 423111; Fax: +9611615021

E-mail: ziad@khoueir.com

Received: 25 September 2014

Accepted in revised form: 23 February 2015

Published online:

1 May 2015 
The following preoperative information was obtained on each patient: sex, age, side of injured eye, time between injury and presentation, time between injury and primary repair, type of injury, pellet entry and exit or impact sites, best corrected visual acuity (BCVA) at presentation, lens status, intraocular pressure (IOP) and extent of posterior segment injury.

Data related to the surgeries included time between primary repair and the first VR procedure, type of anaesthesia, need for concurrent cataract removal, pellet removal, retinopexy to entry and exit wounds, usage of a tamponading agent and/or buckle, antibiotic treatment, postoperative complications and the need for further VR surgery. BCVA and IOP at the last follow-up and the length of the follow-up were retrieved. Complications and reasons for subnormal postoperative visual acuity were also determined

IOL power calculations were based on axial length and keratometry measurements taken on the fellow eye if the measurements on the injured eye were deemed unreliable or not possible. In cases that required circumferential scleral buckling, we subtracted a two dioptres value from the IOL power obtained in order to compensate for the estimated buckle-induced $1 \mathrm{~mm}$ increase in axial length. ${ }^{19}$

\section{Results}

Twenty eyes of 19 patients (all male) with a mean age of 36.1 years (range 16-60 years) were included in the study. Mean postoperative follow-up was 47.5 months (range 15-118 months). Preoperative data are summarised in Table 1.

The time between injury and presentation to our unit ranged between 0 and 37 days with a mean of 1.5 days.
BCVA at presentation ranged from 20/200 to light perception. Entry sites were corneal in eight eyes, limbal in eight eyes and scleral in four eyes. At the time of presentation, 11 eyes had a cataract and 5 eyes had a hyphaema and IOP measurements ranged from 0 to 20 with an average of $5.4 \mathrm{~mm} \mathrm{Hg}$. Preoperative posterior segment visualisation was not possible because of media opacities in 18 eyes. Ten eyes had a penetrating injury and 10 others had a perforating injury with the intraocular pellet impact or exit site being within the arcades in 9 cases and outside the arcades in 11 cases, as determined per-operatively. Three eyes had a retinal detachment at presentation.

All eyes underwent an initial primary repair of the entry site wound either on the same day of the injury or the next day with 13 having undergone primary repair elsewhere. Subsequent posterior segment surgery was delayed in all cases except for three eyes where both primary repair and vitrectomy were performed simultaneously.

Mean time from injury to the first VR procedure was 16.6 days (range 0-64 days). All cases underwent 20-gauge vitrectomy under general anaesthesia. Concurrent lensectomy was performed in 12 eyes of which 2 received an intraocular lens implant.

The intraocular pellet was removed in all the 10 penetrating injuries and retinopexy (cryoptherapy or argon laser) was applied to all eyes with scleral entry wound and to all the impact sites or exit wounds posteriorly.

All the cases received either an intraocular tamponade (silicone oil in 12 cases and sulphur hexafluoride gas (SF6) in 3 cases) or an encircling scleral buckle (276 buckle in two cases and 240 band in 10 cases) and seven cases

Table 1 Preoperative data

\begin{tabular}{|c|c|}
\hline Number of cases & 19 Patients, 20 eyes \\
\hline Demographics & $\begin{array}{l}\text { All males } \\
\text { Average } 31.6 \text { years }(16-60)\end{array}$ \\
\hline Side & $7 \mathrm{OD}, 11 \mathrm{OS}, 1 \mathrm{OU}$ \\
\hline Days from injury to presentation & Median 1.5 days, $(0-37)$ \\
\hline Days from injury to primary repair & 10 Same day, 9 next day \\
\hline Entry site & 8 Corneal, 8 limbal, 4 scleral \\
\hline Type of injury & 10 Penetrating, 10 perforating \\
\hline Penetrating impact site & 5 Macula, 2 juxtamacular, 1 juxtapapillar, 1 equatorial, 1 in scleral wound \\
\hline Perforating exit site & 1 Macula, $1 \mathrm{ONH}, 5$ between arcades and equator, 5 equatorial \\
\hline Visual acuity at presentation & 4 PL, 13 HM, 2 CF, 1 20/200 (PL-20/200) \\
\hline $\mathrm{AC}$ and lens presentation & $\begin{array}{l}5 \text { Hyphaema, } 0 \text { flat } \mathrm{AC} \\
12 \text { Cataract, } 9 \text { clear lens }\end{array}$ \\
\hline IOP at presentation $(\mathrm{mm} \mathrm{Hg})$ & Average 5.45 , median $4.5,(0-20)$ \\
\hline Posterior segment presentation & 18 No view, 1 VH with IOFB, 1 total RRD \\
\hline
\end{tabular}

Abbreviations: AC, anterior chamber; HM, hand motion; IOFB, intraocular foreign body; IOP, intraocular pressure; OD, right eye; ONH, optic nerve head; $\mathrm{OS}$, left eye; OU, both eyes; PL, perception of light; RRD, rhegmatogenous retinal detachment; $\mathrm{VH}$, vitreous haemorrhage. 
had a combination of both internal tamponade and scleral buckle.

All the patients received subconjunctival antibiotic and corticosteroid injections at the end of the surgery and a regimen of topical antibiotics and steroids postoperatively with an additional 1-week course of oral ciprofloxacin given to nine patients.

Complications after the first VR procedure included hypotony (IOP $<7 \mathrm{~mm} \mathrm{Hg}$ ) in one case, silicone oil in the anterior chamber in one case, cataract in two cases, epiretinal membrane (ERM) proliferation at the macula in six cases and rhegmatogenous retinal detachment (RRD) with proliferative vitreoretinopathy (PVR) in five cases. Of these five postoperative RRD cases, three patients had a pre- or per-operative retinal detachment. They had received a SO tamponade in three cases, an encircling 240 band in 1 case and a combination of SO tamponade and a 276 encircling buckle in one case.

A second VR procedure was needed in nine cases. Of these, six cases underwent ERM peeling, with two others undergoing an additional retinectomy and one case undergoing scleral buckling with SF6 injection. The time between the first and second VR procedures ranged between 1.5 and 26 months with a mean of 5.4 months and a median of 3 months. Nine other cases required surgeries for silicone oil removal from the anterior chamber in one eye and from the posterior chamber in four other eyes, phacoemulsification in three eyes and secondary IOL implantation in one eye. Postoperative complications are summarised in Table 2.

Four cases developed complications after the second VR procedure, including ocular hypertension in one eye, cataract in one eye, fibrosis and elevation of the retinectomy edge in one eye and RRD in one eye.

A third VR surgery was performed in two cases for ERM peeling in one eye and lensectomy with SF6 tamponade for the repair of retinal detachment in one other eye. The time between the second and third procedure ranged between 3 weeks and 24 months with an average of 8 months and a median of 5 months.
At last follow-up BCVA ranged from NPL to 20/20 and was $20 / 100$ or better in 10 eyes $(50 \%)$. All the patients had a flat retina except for two cases $(10 \%)$ that developed a retinal detachment complicated by severe proliferative vitreoretinopathy under the silicone oil. Of these, the exit site of the pellet was on the optic nerve head in one case and at the centre of the macula in another case. Both of these cases had one VR procedure only and further surgery was deemed unbeneficial anatomically and functionally.

Five patients were left aphakic (20\%), six cases needed antiglaucoma treatment (33\%) and one case had recurrent uveitis and developed band-shaped keratopathy (5\%).

No cases of endophthalmitis were reported. Pre-, per- and postoperative data are summarised in Table 3.

\section{Discussion}

Pellet gun injuries involving the eyes can be devastating and binocular lesions often occur because of the scattering of the pellets. ${ }^{14}$

Injuries that are limited to the anterior segment can have a good prognosis ${ }^{11,13}$ but penetrating injuries involving the posterior segment are usually more damaging. ${ }^{12,15,20-22}$ These occur when the pellets have enough kinetic energy to go through the anterior segment structures to the posterior segment, resulting in vitreous haemorrhage, direct retinal damage and VR incarceration often progressing to tractional retinal detachments. ${ }^{16}$

Experimental and clinical studies have demonstrated that perforating injuries with posterior segment complications have a poor prognosis especially when treated by conventional techniques. ${ }^{16-18}$ A 5-year survey of ocular shotgun wounds in Ireland published in 1987, shows that seven eyes treated by primary closure alone did not recover useful vision, with five of them developing phthisis, whereas seven other eyes treated by vitrectomy within 1 week of primary closure had a much more favourable visual outcome. ${ }^{15}$

Table 2 Postoperative complications

\begin{tabular}{lll}
\hline $\begin{array}{l}\text { Complications after 1st VR surgery } \\
\text { (20 eyes at an average of } 16.6 \text { days } \\
\text { after injury) }\end{array}$ & $\begin{array}{l}\text { Complications after 2nd surgery (15 } \\
\text { eyes, } 9 \text { VR procedures, at an average of } \\
12.4 \text { months after 1st VR procedure) }\end{array}$ & $\begin{array}{l}\text { Complications after 3rd surgery (2 eyes at an } \\
\text { average of } 8 \text { months after 2nd VR procedure) }\end{array}$ \\
\hline-4 RRD+PVR & $\bullet 1$ Glaucoma & $\bullet 1$ BSK with recurrent anterior uveitis \\
-1 RRD+PVR+cataract & $\bullet 1$ Fibrosis over retinectomy edge & $\bullet 1$ Diplopia \\
-6 Macular ERM & $\bullet 1$ Cataract & \\
-1 Cataract & $\bullet 1$ RRD & \\
-1 Hypotony & & \\
-1 SO in AC & & \\
\hline
\end{tabular}

Abbreviations: AC, anterior chamber; BSK, band-shaped keratopathy; ERM, epiretinal membrane; PVR, proliferative vitreoretinopathy; RRD, rhegmatogenous retinal detachment; SO, silicone oil; VR, vitreoretinal. 


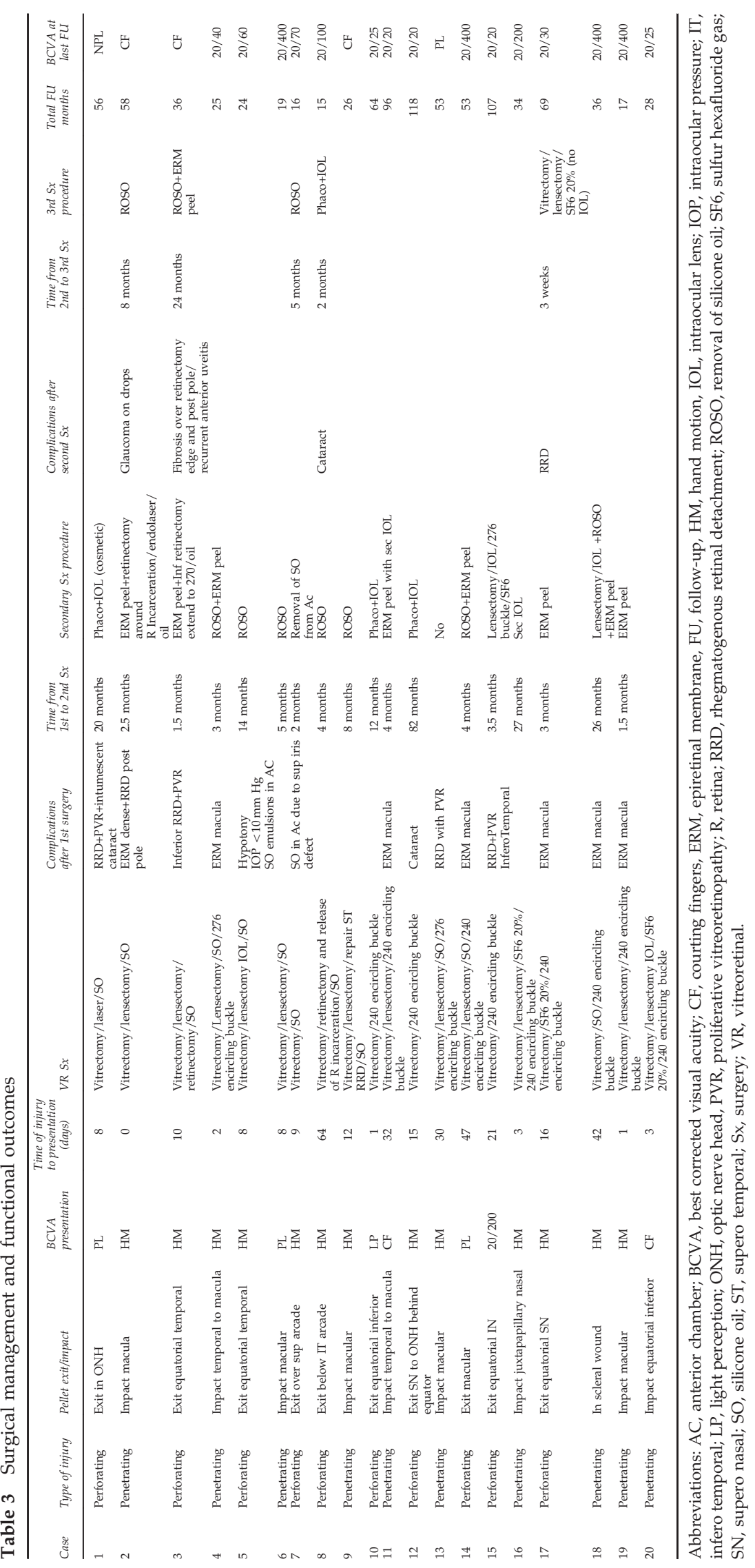


All eyes in our series underwent a 20-gauge vitrectomy at an average of 16.6 days after the trauma and received either an internal tamponade or an encircling buckle or both. Vitrectomy is required to salvage the eye by clearing the vitreous haemorrhage and allowing the removal of the vitreous scaffolding on which the contractile fibroblasts can proliferate and thus aborting and repairing any associated detachment. Chorioretinectomy is sometimes used in selected cases of choroidal impact or perforation $^{23}$ but this techniques was not routinely used by any of the two surgeons involved in the study

Nine cases required one additional VR procedure and two cases required a third VR procedure for the management of postoperative complications. This suggests that eyes with a similar type of injury may require multiple surgical procedures to achieve a desired anatomical and functional outcome in addition to the medical management of other complications such as glaucoma, uveitis or keratopathy. Scar tissue that required reoperation after the first VR surgery was a combination of inferior proliferative vitreoretinopathy and epiretinal membrane formation in the posterior pole. The rate of PVR following the first VR procedure was 25\% (5 eyes out of 20). This rate is comparable to the findings of the eye injury vitrectomy study in which $31.8 \%$ of the eyes with IOFB developed PVR after vitrectomy but the nature of the IOFB was not specified. ${ }^{24}$

All our patients except two, had a flat retina at last follow-up with none of them developing phthisis bulbi. This good anatomical outcome relative to the severity of the posterior segment injury may be related to the timing of the first VR procedure and to the improvement in microsurgical techniques and instrumentation for the management of penetrating and perforating posterior segment injuries. Our results also suggest that for as long as the primary repair is done as early as possible there is no harm in delaying the first vitrectomy surgery in order to decrease the risk of bleeding from the exit wound and to allow for a posterior vitreous detachment to occur.

In terms of functional outcome, the final visual acuity in our patients was related to where the pellet had its impact site on the retina or exit site from the globe, confirming findings from other studies. ${ }^{15}$ All 10 patients who had a final visual acuity of 20/100 or better had an impact or exit site away from the optic nerve or macula (Figure 1). In contrast, all six cases that sustained direct injury the macula or optic nerve head had a final visual acuity of 20/400 or less (Figure 2) as demonstrated by one case in our series where the postoperative visual acuity was NPL due to the location of pellet exit site at the optic nerve head. Alfaro et $a^{25}$ also reported that in patients who achieved visual success after pellet gun injuries, exit wounds outside the vascular arcades were more likely to

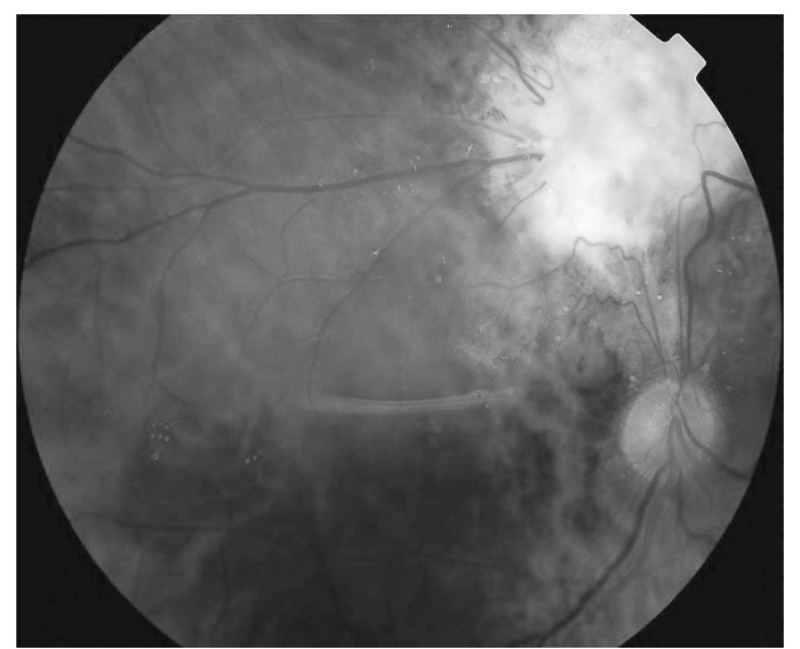

Figure 1 Postoperative fundus photo of a patient with a perforating pellet injury along the superotemporal arcade.

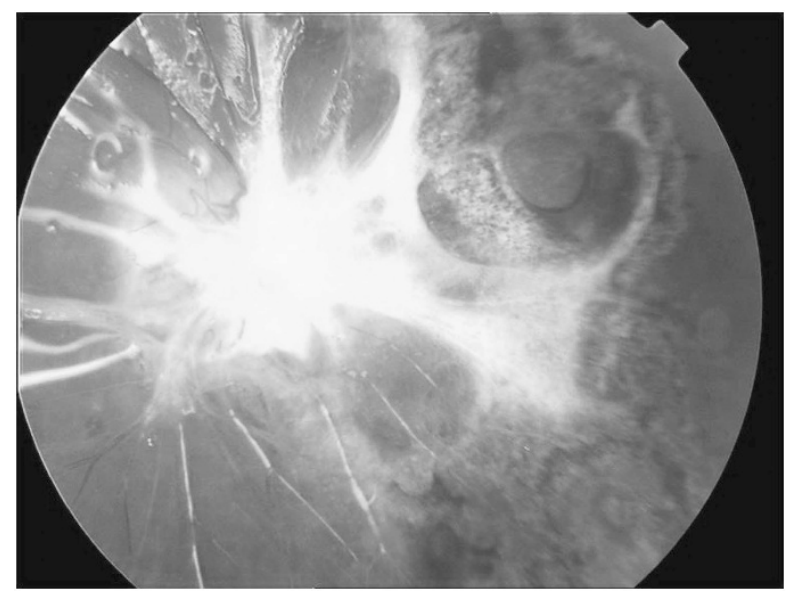

Figure 2 Postoperative fundus photo of a patient with a pellet exit site over the optic nerve head associated with PVR under silicone oil.

be associated with final visual acuities of, or better than, 20/70 than were those within the arcades.

The shape and mass of the intraocular foreign body (IOFB) are germane to the anatomical and functional outcomes. Potts and Dristler observed that the shape of the IOFB affects ocular penetration. ${ }^{26}$ According to their findings, penetration was most difficult for the blunt tip and least difficult for the knife-shaped tip. The pellets in our study were all spherical and the high incidence of posterior segment injuries and penetration would be imputable to their velocity. Woodcock et $a l^{27}$ concluded that IOFBs of greater mass were associated with worse outcomes and if the IOFB reached the posterior segment, its mass is likely to be significantly higher (mean $58 \mathrm{mg}$ ). In their study, $47 \%$ of IOFB were blade shaped and greater mass (mean $122 \mathrm{mg}$ ) was also associated with visual acuity $\leq 20 / 200$ at final follow-up. In our series, although 
we did not measure nor weigh the pellets extracted, we know that they were used for small-bird hunting and they are estimated to be on average of the standard $2.4 \mathrm{~mm}$ calibre weighing $\sim 80 \mathrm{mg}$ per unit. ${ }^{28}$ Their relatively low mass and shape may also account for the good final functional and anatomical outcomes in our patients.

No cases of endophthalmitis were reported in our series. Previous studies have suggested that shotgun wounds are prone to contamination by infectious agents ${ }^{29}$ and that bacteria can survive high-velocities bullets. ${ }^{30,31}$ In our series, the intraocular pellet was removed during vitrectomy in all the penetrating injuries and we used postoperative topical antibiotics in all cases and prophylactic systemic ciprofloxacin in nine patients $(45 \%)$. The use of systemic antibiotics was left to the discretion of the operating surgeon. Only one of them (AA) used systemic antibiotics perioperatively. The number of patients in our study is too small to draw meaningful conclusions on antibiotics use. Other large studies recommending the use of postoperative systemic levofloxacin in the management of patients with perforating/penetrating eye injuries with foreign bodies. ${ }^{32}$ The fact that none of the eyes in our series developed endophthalmitis may also be due to the fact that all the eyes underwent primary repair within 1 (10 eyes) or 2 days (10 eyes) of the penetrating injury. No intraocular or oral steroids were used in the perioperative stage as their role in the management of ocular trauma remains controversial and their effectiveness remains still unproven.

On the other hand, it is important to note that none of the subjects in our study was wearing eye protection. Earlier reports have insisted on the severity of this type of trauma and the necessity to reduce their incidence by publicising their risk and encouraging prophylactic measure such as eye protection. . $^{12,14}$

In conclusion, our results suggest that VR surgery can offer good visual rehabilitation in patients with shotgun eye injuries. The timing and technique of the primary repair and subsequent need for multiple VR procedures may directly affect their anatomical and functional outcome.

\section{Summary}

What was known before

- Eyes with a similar type of injury have a very poor prognosis if left untreated or managed by conventional surgery only. The final visual acuity is related to where the pellet has its impact site on the retina or exit site from the globe.

What this study adds

- Multiple surgical procedures are needed to achieve the desired anatomical and functional outcome. Primary repair should be done as early as possible and delaying the first VR procedure might not be unfavourable to the final outcome.

\section{Conflict of interest}

The authors declare no conflict of interest.

\section{References}

1 Shuttleworth GN, Galloway P, Sparrow JM, Lane C. Ocular air gun injuries: a one-year surveillance study in the UK and Eire (BOSU). 2001-2002. Eye (Lond) 2009; 23(6): 1370-1376.

2 Ramstead C, Ng M, Rudnisky CJ. Ocular injuries associated with Airsoft guns: a case series. Can J Ophthalmol 2008; 43(5): 584-587.

3 McGwin G Jr, Hall TA, Xie A, Owsley C. Gun-related eye injury in the United States, 1993-2002. Ophthalmic Epidemiol 2006; 13(1): 15-21.

4 O'Neill PJ, Lumpkin MF, Clapp B, Kopelman TR, Matthews MR, Cox JC et al. Significant pediatric morbidity and mortality from intracranial ballistic injuries caused by nonpowder gunshot wounds. A case series. Pediatr Neurosurg 2009; 45(3): 205-209.

5 Smith D, Wrenn K, Stack LB. The epidemiology and diagnosis of penetrating eye injuries. Acad Emerg Med 2002; 9 (3): 209-213.

6 Coskunseven E, Onder M, Kymionis GD, Diakonis VF, Arslan E, Tsiklis $\mathrm{N}$ et al. Combined Intacs and posterior chamber toric implantable Collamer lens implantation for keratoconic patients with extreme myopia. Am J Ophthalmol 2007; 144(3): 387-389.

7 Chen AY, Stewart MG, Raup G. Penetrating injuries of the face. Otolaryngol Head Neck Surg 1996; 115(5): 464-470.

8 Kratz A, Levy J, Cheles D, Ashkenazy Z, Tsumi E, Lifshitz T. Airsoft gun-related ocular injuries: novel findings, ballistics investigation, and histopathologic study. Am J Ophthalmol 2010; 149(1): 37-44.

9 Pulido JS, Gupta S, Folk JC, Ossoiny KC. Perforating BB gun injuries of the globe. Ophthalmic Surg Lasers 1997; 28(8): 625-632.

10 Bowen DI, Magauran DM. Ocular injuries caused by airgun pellets: an analysis of 105 cases. Br Med J 1973; 1(5849): 333-337.

11 Colyer MH, Chun DW, Bower KS, Dick JSB, Weichel ED. Perforating globe injuries during operation Iraqi Freedom. Ophthalmology 2008; 115(11): 2087-2093.

12 Fournier D, Cherifi M, Salvanet-Bouccara A, Forestier F, Feys J. [Eye injuries due to pellet guns. Apropos of 7 cases]. Ophtalmologie 1989; 3(4): 303-305.

13 Finkelstein M, Legmann A, Rubin PA. Projectile metallic foreign bodies in the orbit: a retrospective study of epidemiologic factors, management, and outcomes. Ophthalmology 1997; 104(1): 96-103.

14 Assaf E, Emadisson H, Bendeddouche K, Forestier F, Salvanet-Bouccara A. [Pellet guns: a persistent threat to eyes]. J Fr Ophtalmol 2003; 26(9): 960-966.

15 Roden D, Cleary P, Eustace P. A five-year survey of ocular shotgun injuries in Ireland. Br J Ophthalmol 1987; 71(6): 449-453.

16 Cleary PE, Ryan SJ. Method of production and natural history of experimental posterior penetrating eye injury in the rhesus monkey. Am J Ophthalmol 1979; 88(2): 212-220.

17 Cleary PE, Ryan SJ. Histology of wound, vitreous, and retina in experimental posterior penetrating eye injury in the rhesus monkey. Am J Ophthalmol 1979; 88(2): 221-231. 
18 Michels RG. Vitreous Surgery. Am Acad Ophthalmol 1982; $126 \mathrm{p}$.

19 Smiddy WE, Loupe DN, Michels RG, Enger C, Glaser BM, deBustros S. Refractive changes after scleral buckling surgery. Arch Ophthalmol 1989; 107(10): 1469-1471.

20 Noye JF, Bernhard F, Berrod JP, Lesure P. [Penetrating eye injuries caused by firearms. Apropos of 17 cases]. Bull Soc Ophtalmol Fr 1989; 89(4): 629-632.

21 Boscher-Southall C, Manderieux N, Konqui P, Pouliquen Y. [Eye injuries caused by lead shot or hunting shot. Apropos of 38 cases]. Bull Soc Ophtalmol Fr 1988; 88(2): 195-200.

22 Roussat B, Ruellan YM, Leroy C. [Injuries caused by BB guns. Clinical, therapeutic and medico-legal aspects]. Bull Soc Ophtalmol Fr 1986; 86(10): 1119-1122.

23 Weichel ED, Bower KS, Colyer MH. Chorioretinectomy for perforating or severe intraocular foreign body injuries. Graefes Arch Clin Exp Ophthalmol 2010; 248(3): 319-330.

24 Feng $\mathrm{K}, \mathrm{Hu}$ Y, Wang C, Shen L, Pang X, Jiang Y et al. Risk factors, anatomical, and visual outcomes of injured eyes with proliferative vitreoretinopathy: eye injury vitrectomy study. Retina 2013; 33(8): 1512-1518.
25 Alfaro DV, Tran VT, Runyan T, Chong LP, Ryan SJ, Liggett PE. Vitrectomy for perforating eye injuries from shotgun pellets. Am J Ophthalmol 1992; 114(1): 81-85.

26 Potts AM, Distler JA. Shape factor in the penetration of intraocular foreign bodies. Am J Ophthalmol 1985; 100(1): 183-187.

27 Woodcock MGL, Scott RAH, Huntbach J, Kirkby GR. Mass and shape as factors in intraocular foreign body injuries. Ophthalmology 2006; 113(12): 2262-2269.

28 GunsOnPegs [Internet]. Eley Cartridge Information. Available from http://www.gunsonpegs.com/shooting/ buy/cartridge_information.php\#shotsize.

29 Kara MI, Polat HB, Ay S. Penetrated shotgun pellets: a case report. Eur J Dent 2008; 2: 59-62.

30 Wolf AW, Benson DR, Shoji H, Hoeprich P, Gilmore A. Autosterilization in low-velocity bullets. J Trauma 1978; 18(1): 63.

31 Mastrapa RME, Glanzberg H, Head JN, Melosh HJ, Nicholson WL. Survival of bacteria exposed to extreme acceleration: implications for panspermia. Earth Planet Sci Lett 2001; 189(1): 1-8.

32 Colyer MH, Weber ED, Weichel ED, Dick JSB, Bower KS, Ward TP et al. Delayed intraocular foreign body removal without endophthalmitis during Operations Iraqi Freedom and Enduring Freedom. Ophthalmology 2007; 114(8): 1439-1447. 